\title{
Plastics disassembly versus bulk recycling: engineering design for end-of-life electronics resource recovery
}

\author{
Pedro Rios ${ }^{1}$, Julie Ann Stuart ${ }^{1 *}$, Ed Grant ${ }^{2}$ \\ ${ }^{1}$ School of Industrial Engineering \\ Purdue University \\ prios@purdue.edu, stuart@purdue.edu \\ ${ }^{2}$ Department of Chemistry \\ Purdue University \\ edgrant@purdue.edu
}

\section{Supporting Information}

Contact Author Information:

Julie Ann Stuart, Ph.D., P.E.

Assistant Professor

Purdue University

School of Industrial Engineering

Grissom Hall Room 258

315 N. Grant Street

West Lafayette, IN 47907-2023

EMAIL: stuart@purdue.edu

TEL: 765-494-6256

FAX: 765-494-1299 


\section{TABLE S1a: Truckload Compositions and Distributions for Interarrival Times for CPM (computers, printers and monitors) (1)}

\begin{tabular}{|c|c|c|c|c|c|c|c|}
\hline \multirow[b]{2}{*}{ Collection Pilot Arrival } & \multicolumn{5}{|c|}{ Product Type } & \multirow[b]{2}{*}{$\begin{array}{r}\frac{\text { Total }}{\text { Weight }} \\
\underline{\text { kg/Arrival }}\end{array}$} & \multirow[b]{2}{*}{$\frac{\begin{array}{r}\text { Distribution for } \\
\text { Interarrival Time }\end{array}}{\underline{\text { (weeks) }}}$} \\
\hline & Arrival \# & $\underline{\text { TVs }}$ & $\underline{\text { PCs }}$ & Monitors & $\underline{\text { Printers }}$ & & \\
\hline NY, Binghamton Fall 1996 & Arrival 1 & -- & 7 & 8 & 2 & 206 & $\operatorname{Exp}(1)$ \\
\hline NY, Binghamton Spring 1997 & Arrival 2 & -- & 19 & 33 & 9 & 753 & $\operatorname{Exp}(1)$ \\
\hline NY, Somerville Fall 1996 & Arrival 3 & -- & 21 & 17 & 12 & 569 & $\operatorname{Exp}(1)$ \\
\hline NY, Somerville Spring 1997 & Arrival 4 & -- & 72 & 52 & 40 & 1,852 & $\operatorname{Exp}(1)$ \\
\hline MN, Hennepin County 1995 & Arrival 5 & -- & 67 & 673 & 189 & 11,996 & $\operatorname{Exp}(12)$ \\
\hline MN, Hennepin County 1996 & Arrival 6 & -- & 661 & 1,156 & 261 & 25,874 & $\operatorname{Exp}(12)$ \\
\hline MN, Hennepin County 1997 & Arrival 7 & -- & 1,331 & 1,734 & 554 & 43,793 & $\operatorname{Exp}(12)$ \\
\hline IL, Naperville 1996 & Arrival 8 & -- & 367 & 152 & 113 & 7,045 & $\operatorname{Exp}(2)$ \\
\hline IL, Naperville 1997 & Arrival 9 & -- & 305 & 290 & 130 & 8,515 & $\operatorname{Exp}(2)$ \\
\hline IL, Wheaton 1998 & Arrival 10 & -- & 99 & 226 & 102 & 5,167 & $\operatorname{Exp}(2)$ \\
\hline
\end{tabular}

$\operatorname{Exp}(\lambda)$ represents an exponentially distributed random variable with mean $\lambda$.

\section{TABLE S1b: Truckload Compositions and Distributions for Interarrival Times for T-CPM (televisions, computers, printers, and monitors) (1)}

\begin{tabular}{|c|c|c|c|c|c|c|c|}
\hline \multirow[b]{2}{*}{ Collection Pilot Arrival } & \multicolumn{5}{|c|}{ Product Type } & \multirow[b]{2}{*}{ 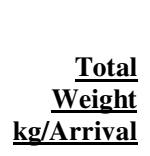 } & \multirow[b]{2}{*}{$\frac{\begin{array}{l}\text { Distribution for } \\
\text { Interarrival Time }\end{array}}{\text { (weeks) }}$} \\
\hline & $\underline{\text { Arrival \# }}$ & $\underline{\text { TVs }}$ & $\underline{\text { PCs }}$ & $\underline{\text { Monitors }}$ & $\underline{\text { Printers }}$ & & \\
\hline NY, Binghamton Fall 1996 & Arrival 1 & 23 & 7 & 8 & 2 & 841 & $\operatorname{Exp}(1)$ \\
\hline NY, Binghamton Spring 1997 & Arrival 2 & 52 & 19 & 33 & 9 & 2,187 & $\operatorname{Exp}(1)$ \\
\hline NY, Somerville Fall 1996 & Arrival 3 & 54 & 21 & 17 & 12 & 2,058 & $\operatorname{Exp}(1)$ \\
\hline NY, Somerville Spring 1997 & Arrival 4 & 61 & 72 & 52 & 40 & 3,534 & $\operatorname{Exp}(1)$ \\
\hline MN, Hennepin County 1995 & Arrival 5 & 4,428 & 67 & 673 & 189 & 134,101 & $\operatorname{Exp}(12)$ \\
\hline MN, Hennepin County 1996 & Arrival 6 & 5,115 & 661 & 1,156 & 261 & 166,925 & $\operatorname{Exp}(12)$ \\
\hline MN, Hennepin County 1997 & Arrival 7 & 7,376 & 1,331 & 1,734 & 554 & 247,192 & $\operatorname{Exp}(12)$ \\
\hline IL, Naperville 1996 & Arrival 8 & 111 & 367 & 152 & 113 & 10,106 & $\operatorname{Exp}(2)$ \\
\hline IL, Naperville 1997 & Arrival 9 & 292 & 305 & 290 & 130 & 16,568 & $\operatorname{Exp}(2)$ \\
\hline IL, Wheaton 1998 & Arrival 10 & 102 & 99 & 226 & 102 & 7,980 & $\operatorname{Exp}(2)$ \\
\hline
\end{tabular}

$\operatorname{Exp}(\lambda)$ represents an exponentially distributed random variable with mean $\lambda$. 
TABLE S2: Average Product Material Composition, Weight, and Revenue (2, 3)

\begin{tabular}{|c|c|c|c|c|c|c|c|}
\hline \multirow{2}{*}{ Material } & \multirow[b]{2}{*}{$(\$ / \mathrm{kg})$} & \multicolumn{6}{|c|}{ Product Type } \\
\hline & & $\underline{\text { TVs }}$ & $\underline{\mathrm{PCs}}$ & Monitors & Printers & PC-P & $\underline{\mathrm{PC}-\mathrm{M}}$ \\
\hline Glass & $\$ 0.12$ & $31.5 \%$ & $0.0 \%$ & $37.0 \%$ & $0.0 \%$ & $0.0 \%$ & $0.0 \%$ \\
\hline Mixed plastic & $\$ 0.04$ & $1.5 \%$ & $0.2 \%$ & $2.1 \%$ & $5.8 \%$ & $0.3 \%$ & $1.3 \%$ \\
\hline Plastic & $\$ 0.63$ & $13.8 \%$ & $4.3 \%$ & $18.7 \%$ & $23.0 \%$ & $5.7 \%$ & $0.0 \%$ \\
\hline Mixed metal & $\$ 0.22$ & $13.0 \%$ & $50.4 \%$ & $20.4 \%$ & $55.1 \%$ & $48.9 \%$ & $53.6 \%$ \\
\hline Ferrous metal & $\$ 0.04$ & $25.5 \%$ & $45.1 \%$ & $20.8 \%$ & $15.6 \%$ & $45.1 \%$ & $45.1 \%$ \\
\hline Other & $\$(0.04)$ & $14.7 \%$ & $0.0 \%$ & $1.0 \%$ & $0.5 \%$ & $0.0 \%$ & $0.0 \%$ \\
\hline & Weight (Kg) & 27.58 & 10.64 & 13.26 & 8.43 & 10.85 & 10.20 \\
\hline Average Reven & Product Type & $\$ 4.41$ & $\$ 1.68$ & $\$ 2.89$ & $\$ 2.32$ & $\$ 1.77$ & $\$ 1.41$ \\
\hline
\end{tabular}

PC-P and PC-M represent computers with plastic and metal covers respectively.

TABLE S3: Distributions for Disassembly Processing Times and Disassembly Scheduling Rank for SDT (Space per disassembly time ratio) (3-5)

\begin{tabular}{ccccc} 
Product Type & Average size $\left(\mathrm{m}^{3}\right)$ & $\begin{array}{c}\text { Pre-Disassembly Planning } \\
\text { and Disassembly Time (minutes) }\end{array}$ & SDT Rank \\
\cline { 2 - 3 } TVs & 0.1650 & & $\operatorname{Logn}(2.9,1.5)$ & 1 \\
Monitors & 0.0651 & $\operatorname{Logn}(1.4,1.5)$ & 2 \\
Printers & 0.0397 & $\operatorname{Logn}(2.5,1.6)$ & 4 \\
PC-P & 0.0312 & $\operatorname{Logn}(1.3,0.58)$ & 3 \\
\hline
\end{tabular}

$\operatorname{Logn}\left(\mu, \sigma^{2}\right)$ represents a Lognormally distributed random variable with mean $\mu$ and variance $\sigma^{2}$. PC-P represents computers with plastic covers.

\title{
TABLE S4: Number of plastic pieces for identification and plastic cover composition (3)
}

\author{
Product Family \\ Televisions \\ Computers \\ Monitors \\ Printers
}

Average \# of Plastic Pieces
1
2
1
3


TABLE S5: Plastic Identification Process Steps and Time (3)

\begin{tabular}{|c|c|c|c|c|c|}
\hline \multirow{2}{*}{$\begin{array}{c}\text { Raman } \\
\text { spectrochemical } \\
\underline{\text { identification }}\end{array}$} & \multicolumn{3}{|c|}{ Process steps } & \multirow{2}{*}{$\begin{array}{l}\text { Process } \\
\text { time } \\
(\mathrm{sec})\end{array}$} & \multirow{2}{*}{$\begin{array}{l}\text { Identification } \\
\text { success } \\
\text { percentage of } \\
\text { total flow }\end{array}$} \\
\hline & $\begin{array}{c}\text { Material } \\
\text { handling setup }\end{array}$ & $\begin{array}{l}\text { Laser read } \\
\text { number }\end{array}$ & Sortation & & \\
\hline First-entrant flow & $\checkmark$ & 1 & $\checkmark$ & $\overline{5.4}$ & $80 \%$ \\
\hline of plastic pieces & $\checkmark$ & 2 & $\checkmark$ & 7.1 & $10 \%$ \\
\hline & $\checkmark$ & 3 & $\checkmark$ & 8.7 & $9.5 \%$ \\
\hline $\begin{array}{l}\text { Re-entrant flow } \\
\text { of plastic pieces }\end{array}$ & $\checkmark$ & 3 & $\checkmark$ & 11 & $0.25 \% *$ \\
\hline
\end{tabular}

\section{TABLE S6: Binary Designation for Changeovers at Product Shredding}

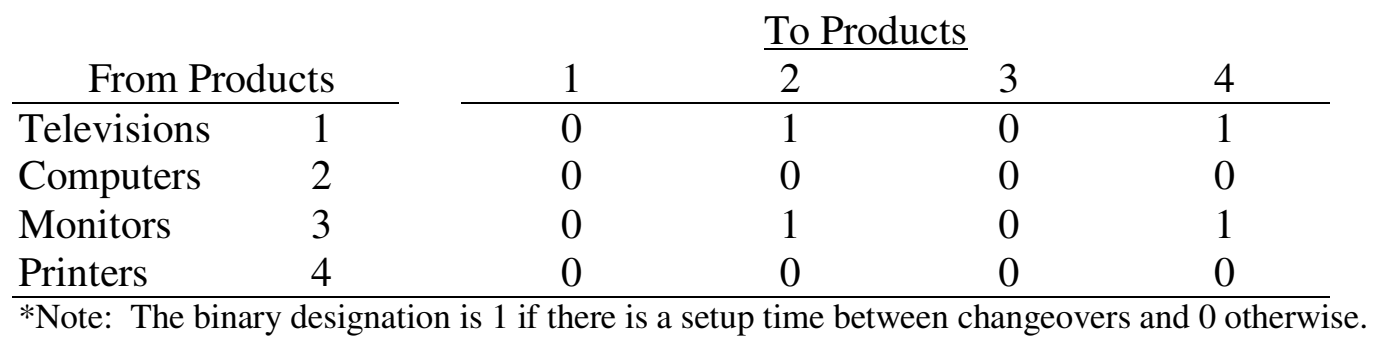

\section{TABLE S7: Binary Designation for Changeovers at Polymer Shredding}

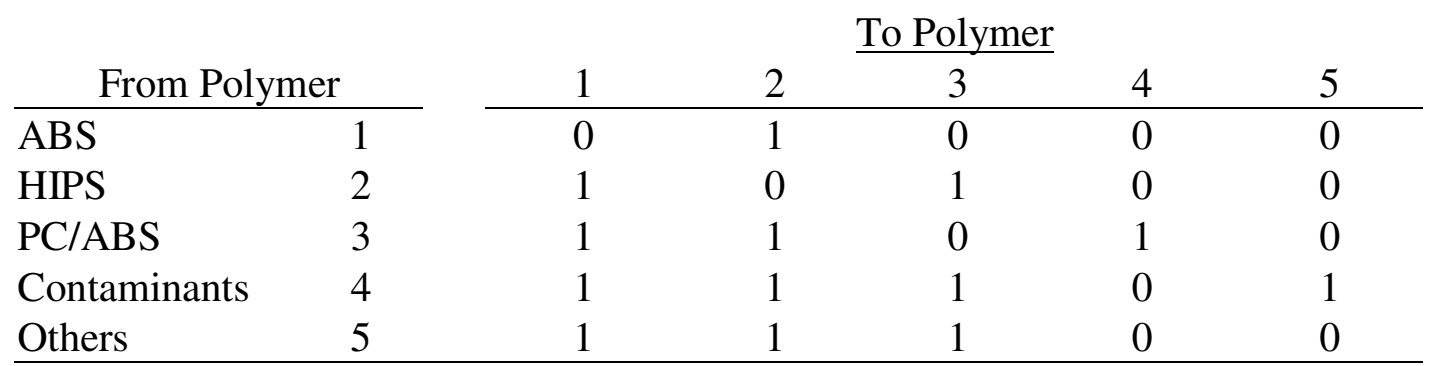

*Note: The binary designation is 1 if there is a setup time between changeovers and 0 otherwise. 


\section{TABLE S8(a): Input Statistics for CPM Arrival Quantities at Confidence Level 95\%}

\begin{tabular}{|c|c|c|c|c|c|c|c|c|c|c|}
\hline \multirow[b]{2}{*}{$\underline{\text { Scenario }}$} & \multicolumn{2}{|c|}{$\underline{\mathrm{TV}}$} & \multicolumn{2}{|c|}{$\underline{\mathrm{PC}-\mathrm{M}}$} & \multicolumn{2}{|c|}{$\underline{\mathrm{PC}-\mathrm{P}}$} & \multicolumn{2}{|c|}{ Monitor } & \multicolumn{2}{|c|}{ Printer } \\
\hline & mean & $\begin{array}{l}\text { half } \\
\text { width }\end{array}$ & mean & $\begin{array}{l}\text { half } \\
\text { width }\end{array}$ & mean & $\begin{array}{l}\text { half } \\
\text { width }\end{array}$ & mean & $\begin{array}{l}\text { half } \\
\text { width }\end{array}$ & mean & $\frac{\text { half }}{\text { width }}$ \\
\hline Bulk/CPM & 0 & 0 & 12,137 & 292 & 23,539 & 573 & 39,214 & 1,104 & 16,783 & 372 \\
\hline Disassembly/CPM & 0 & 0 & 12,079 & 262 & 23,441 & 510 & 39,198 & 1,040 & 16,793 & 325 \\
\hline
\end{tabular}

TABLE S8(b): Input Statistics for T-CPM Arrival Quantities at Confidence Level 95\%

\begin{tabular}{lccccccccccccc} 
& \multicolumn{2}{c}{$\underline{\text { TV }}$} & \multicolumn{2}{c}{ PC-M } & & \multicolumn{2}{c}{$\underline{\text { PC-P }}$} & \multicolumn{2}{c}{ Monitor } & \multicolumn{2}{c}{ Printer } \\
Scenario & $\underline{\text { mean }}$ & $\underline{\text { width }}$ & & $\underline{\text { mean }}$ & $\underline{\text { width }}$ & $\underline{\text { mean }}$ & $\underline{\text { width }}$ & $\underline{\text { mean }}$ & $\underline{\text { width }}$ & $\underline{\text { mean }}$ & $\underline{\underline{\text { hidth }}}$ \\
Bulk/T-CPM & 99,604 & 4,752 & 12,137 & 292 & 23,539 & 573 & 39,214 & 1,104 & 16,783 & 372 \\
Disassembly/T-CPM & 92,429 & 3,927 & 11,810 & 256 & 22,919 & 506 & 37,679 & 971 & 16,398 & 331 \\
\hline
\end{tabular}

Note: The 95\% confidence interval is constructed by adding and subtracting the half-width from the mean.

\section{References}

(1) Analysis of Five Community Consumer/Residential Collections of End-of-Life

Electronic and Electrical Equipment. Tech. Report No. EPA-901-R-98-003.

United Stated Environmental Protection Agency Region 1, Common Sense

Initiative Computer and Electronic Sector, 1999.

(2) Residential Collection of Household End-of-Life Electrical and Electronic

Equipment: Pilot Collection Project” Tech. Report No. EPA-901-E-98-002.

United Stated Environmental Protection Agency Region 1, Common Sense

Initiative Computer and Electronic Sector, 1998.

(3) J. A. Stuart et al., Purdue University, unpublished results, 2003.

(4) J. A. Stuart, V. Christina, To appear in IEEE Transactions Electronic Packaging

Manufacturing 2003. 
(5) C. B. Boks, E. Kroll, W. C. J. Brouwers, A. L. N. Stevels, Presented at the IEEE Symposium on Electronics and the Environment, Dallas, May 6-8, 1996; pp. 224229. 